Ori gi n of scal ing struct ure and non- Gaussi an vel ocity di stributi on in a sel f-gravitating ring model

\begin{tabular}{|l|l|}
\hline 著者 & $\begin{array}{l}\text { SOTA Yasuhi de, I GUCH Osamu, MORI KAWA } \\
\text { Nasahi ro, TATEKAWA Takayuki, MAEDA Ken- i chi }\end{array}$ \\
\hline $\begin{array}{l}\text { j our nal or } \\
\text { publ i cat i on t i t l e }\end{array}$ & Physi cal revi ew. Thi rd ser i es. E \\
\hline vol ume & 64 \\
\hline page $r$ ange & 56133 \\
\hline year & $2001-10$ \\
\hline URL & ht t p: //hdl . handl e. net /10098/6425 \\
\hline
\end{tabular}




\title{
Origin of scaling structure and non-Gaussian velocity distribution in a self-gravitating ring model
}

\author{
Yasuhide Sota, ${ }^{1,2, *}$ Osamu Iguchi, ${ }^{1, \dagger}$ Masahiro Morikawa, ${ }^{1, \$}$ Takayuki Tatekawa, ${ }^{3,8}$ and Kei-ichi Maeda ${ }^{2,3,4, \|}$ \\ ${ }^{1}$ Department of Physics, Ochanomizu University, 2-1-1 Ohtuka, Bunkyo, Tokyo,112-8610, Japan \\ ${ }^{2}$ Advanced Research Institute for Science and Engineering, Waseda University, Shinjuku, Tokyo 169-8555, Japan \\ ${ }^{3}$ Department of Physics, Waseda University, Shinjuku, Tokyo 169-8555, Japan \\ ${ }^{4}$ Advanced Institute for Complex Systems, Waseda University, Shinjuku, Tokyo 169-8555, Japan
}

(Received 27 September 2000; revised manuscript received 17 April 2001; published 30 October 2001)

\begin{abstract}
Fractal structures and non-Gaussian velocity distributions are characteristic properties commonly observed in virialized self-gravitating systems, such as galaxies and interstellar molecular clouds. We study the origin of these properties using a one-dimensional ring model that we propose in this paper. In this simple model, $N$ particles are moving, on a circular ring fixed in three-dimensional space, with mutual interaction of gravity. This model is suitable for the accurate symplectic integration method by which we argue the phase transition in this system. Especially, in between the extended phase and the collapsed phase, we find an interesting phase (halo phase) that has negative specific heat at the intermediate energy scale. Moreover, in this phase, there appear scaling properties and nonthermal and non-Gaussian velocity distributions. In contrast, these peculiar properties are never observed in other gas and core phases. Particles in each phase have a typical time scale of motions determined by the cutoff length $\xi$, the ring radius $R$, and the total energy $E$. Thus all relaxation patterns of the system are determined by these three time scales.
\end{abstract}

DOI: 10.1103/PhysRevE.64.056133

PACS number(s): 05.70.Fh, 05.45.Df, 05.65.+b, 98.35.Ac

\section{INTRODUCTION}

Many astrophysical objects in our universe consist of mutually interacting elements through gravity. If they are almost isolated systems, they are called self-gravitating system (SGS). For example, galaxies, clusters of galaxies, globular clusters, and molecular clouds are thought to be typical SGSs. Their statistical properties are often characterized by non-Gaussian velocity (or pair-wise velocity) distributions [1], fractal structures [2], and the scaling relation between the mass density and the system size [3]. Most of these objects are thought to be gravitationally virialized. Therefore, pure gravitational force seems to play an essential role in characterizing the above statistical properties of SGS independently of initial conditions.

There have been some theoretical approaches to explain the fractal structures in SGS from the viewpoint of criticality and phase transition in gravothermodynamics [4]. Strictly speaking, the ordinary SGSs in three dimension (3D) cannot attain genuine stable equilibrium, because the gravitational force does not vanish at long distances (IR divergence) and diverges at short distances (UV divergence). These properties of gravity cause gravothermal catastrophe in a selfgravitating gas system enclosed by a solid adiabatic wall. In fact, the isothermal sphere is not always stable, since the entropy does not necessarily take the local maximum for this configuration [5-7]. Therefore, the introduction of a smallscale cutoff as well as a large-scale cutoff is inevitable for such unstable systems to attain the final equilibrium. Though

\footnotetext{
*Email address: sota@ skyrose.phys.ocha.ac.jp

†Email address: osamu@phys.ocha.ac.jp

\#Email address: hiro@phys.ocha.ac.jp

${ }^{\S}$ Email address: tatekawa@ gravity.phys.waseda.ac.jp

"Email address: maeda@mse.waseda.ac.jp
}

the introduction of the cutoff prevents the gravothermal catastrophe, 3D gravitational system has a phase with negative specific heat when treated in the microcanonical ensemble. Even in the canonical ensemble, this system is highly unstable. When the temperature decreases, the system experiences a violent first-order phase transition from the gas phase into the cluster phase $[6,8]$. According to these arguments, no stable equilibrium states are theoretically expected in the 3D gravitational system with or without cutoff in any ensemble.

On the other hand, in the real world, we do not need stable equilibrium states for describing SGS; metastable equilibrium states do actually appear in the dynamical description, such as, collisionless Boltzmann equation. The finite lifetime of such structures is sufficient to explain the present structures of SGS, even if they are expected to evolve further into different quasistable states through twobody relaxation.

The relaxation process of SGS has been mainly discussed in the one-dimensional gravitational sheet model (1DS) $[9,10]$. In this model, many parallel sheets interact with each other through constant force that never decays at distant places. Though the interaction is long ranged, no phase transition occurs in 1DS. Thermodynamics of 1DS is exactly solved and actually, in numerical calculations, the system reaches thermal equilibrium long after it attains virial equilibrium. In this model, the virial condition gives the relation $2\langle K\rangle=\langle V\rangle$ between the time averaged kinetic energy $\langle K\rangle$ and the potential energy $\langle V\rangle$. Therefore, contrary to the 3D SGS, specific heat of 1DS is always positive. Thus the relaxation process in 1DS would be quite different from that in 3D SGS. Another well-known one-dimensional model that has long-range force is the Hamilton mean-field (HMF) model, in which phase transition does occur [11]. There have been a lot of studies on the relaxation process of HMF. Actually, Lévy-type jumping motion of constituent particles 
and superdiffusion process have been revealed in HMF [12]. In this HMF, though the specific heat becomes negative in the quasistable state [13], it remains positive in the thermal equilibrium state and the phase transition turns out to be of the second order. Despite some common properties of the models, the interaction form in HMF is also quite different from that of real 3D gravitational systems.

Several other simple models have been proposed [14] in order to characterize the 3D SGS much faithfully. For example, the cell model and its extended versions are the simplest models that show phase transition. In these models, the pair interaction potential changes its value only at the cell boundary $[15,16]$. At low temperatures, most particles are trapped within several clusters, and at higher temperatures, these clusters melt and the particles can move freely. However, it seems difficult to examine the relaxation process of this system numerically, since the interaction is not efficient to cause sufficient relaxation; particles have no interaction within each cell. There are also several numerical analyses with the different type interactions including the lower and higher cutoffs $[16,17]$. However, the value of the lower cutoff used in realistic numerical calculations of these models seems to be too large to extract the intrinsic properties of realistic 3D SGS.

Thus it seems indispensable for us to have a model that faithfully reflects the characteristic properties of 3D SGSs; the model has negative specific heat and shows a phase transition similar to 3D SGSs, and is capable for us to analyze quasiequilibrium states that would be realized before the system reaches the complete equilibrium.

In this paper, therefore, we first propose a model (Selfgravitating ring: SGR) that (a) has negative specific heat, (b) shows phase transition representing 3D SGS, and (c) is numerically tractable. This model consists of $N$ particles, which can freely move on a ring with a fixed radius, mutually interacting through 3D gravity. An excellent point of this model is that the force is genuine 3D gravity while the calculation is essentially one dimensional. Moreover, the Hamiltonian permits the accurate symplectic integration method by which we can analyze the nature of quasiequilibrium states and phase transitions within very long time scales in this model.

In Sec. II, we introduce the SGR model, and in Sec. III identify three quasiequilibrium states including the state with negative specific heat. In Sec. IV, we analyze the particle motions from a statistical point of view, and then we study the relaxation process toward the thermodynamical equilibrium in Sec. V, and the scaling structure in Sec. VI. Finally, we discuss our results in Sec. VII.

\section{SELF-GRAVITATING RING MODEL}

In this section, we introduce the SGR model, in which particles interact with each other through genuine 3D gravity while the particle motion is constrained on a $1 \mathrm{D}$ ring. By utilizing this model, we study the gravitational phase transition, which was not feasible in the 1DS model [10]. The lack of phase transition in 1DS is related to the fact that the potential of 1DS increases linearly without bound, and, there-

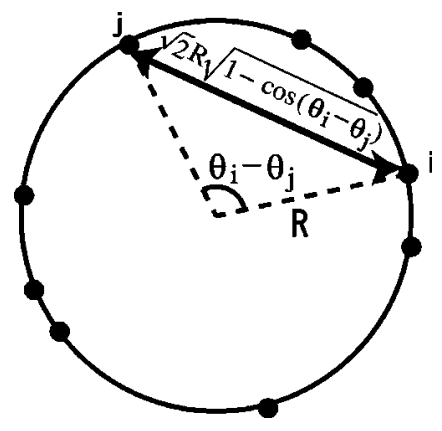

FIG. 1. SGR model with a fixed radius $R$. The particle locations are specified by the angles measured from a fixed direction. A pair of particles at $\theta_{i}$ and at $\theta_{j}$ interact with each other through the inverse-square law 3D gravitational force; the distance is measured by the straight line in the picture and not by $R\left|\theta_{i}-\theta_{j}\right|$.

fore, there is no characteristic energy scale required for a particle to escape from the cluster. On the other hand, we have the characteristic energy scale to bind particles in our SGR model.

We consider a system of self-gravitating $N$ particles with mass $m$, whose motions are smoothly constrained on a circular ring with a fixed radius $R$ without friction (Fig. 1). Each pair of particles interact with each other through a 3D gravitational force. The distance between a pair is measured by the length of the straight line combining the pair and not by the minor arc of the ring.

The Hamiltonian of this system becomes

$$
H_{p}=\frac{1}{2 m R^{2}} \sum_{i=1}^{N} P_{i}^{2}-\sum_{i<j}^{N} \frac{G m^{2}}{\sqrt{2} R \sqrt{1-\cos \left(\theta_{i}-\theta_{j}\right)+\epsilon}} .
$$

The position of $i$ th particle is fully described by the angular variable $\theta_{i}$ as $\boldsymbol{r}_{i}=\left(R \sin \theta_{i}, R \cos \theta_{i}\right)$. The momentum conjugate to $\theta_{i}$ is given by $P_{i}=m R^{2} d \theta_{i} / d t$. The UV-cutoff parameter $\epsilon$ truncates the diverging gravitational force at around the distance $\xi \equiv \sqrt{2 \epsilon} R$.

We first introduce three dynamical time scales that are apparent in the above Hamiltonian. They are parametrized by the ring radius $R$, the cutoff scale $\xi$, and the total energy $E$ of the system.

When the system is almost uniformly filled by moving particles, we have the longest dynamical time $t_{R}$ defined by

$$
t_{R} \equiv \sqrt{\frac{R^{3}}{G N m}}
$$

During this time $t_{R}$, a typical particle goes around the ring once. Therefore, the time scale for the whole system to attain thermodynamical equilibrium, if any, is at least larger than this time scale.

When all the particles collapse completely into a core, we find the shortest dynamical time scale $t_{\xi}$ defined as

$$
t_{\xi} \equiv \sqrt{\frac{\xi^{3}}{G N m}}
$$


During this time $t_{\xi}$, a typical particle bound to the core oscillates once ${ }^{1}$.

There is an another time scale in between the above two extreme time scales. Suppose the system is stably confined in a region with a scale $r$, which satisfies

$$
\xi \ll r \ll R .
$$

For this scale $r$, the ring can be approximated as an infinite straight line and the cutoff $\xi$ can be neglected. Then the leading term of the denominator of the potential, term in the Hamiltonian (2.1) becomes $\left|\theta_{i}-\theta_{j}\right|$ and

$$
H_{p} \approx \frac{1}{2 m R^{2}} \sum_{i=1}^{N} P_{i}^{2}-\sum_{i<j}^{N} \frac{G m^{2}}{R\left|\theta_{i}-\theta_{j}\right|} .
$$

In this case, the potential term $V_{p}$ satisfies Euler's theorem for homogeneous function

$$
\sum_{i=1}^{N} \theta_{i} \frac{\partial V_{p}}{\partial \theta_{i}}=-V_{p},
$$

and the ordinary virial condition holds

$$
2\left\langle K_{p}\right\rangle=-\left\langle V_{p}\right\rangle,
$$

where angle brackets represent the long-time average. ${ }^{2}$ According to this virial relation, the typical size of a system $r$ is related with the total energy $E$ as

$$
E=\frac{V_{p}}{2} \equiv-\frac{G m^{2} N^{2}}{4 r} .
$$

Hence the above condition for $r$ (2.4) reads

$$
\frac{G m^{2} N^{2}}{4 \xi} \gg|E| \gg \frac{G m^{2} N^{2}}{4 R} .
$$

Moreover from the virial relation, the velocity dispersion is given as

$$
2\left\langle K_{p}\right\rangle=-\left\langle V_{p}\right\rangle=m N\left\langle v^{2}\right\rangle,
$$

where $v \equiv d r / d t$ and, therefore,

$$
\sqrt{\left\langle v^{2}\right\rangle}=\sqrt{\frac{2|E|}{m N}}
$$

From this Eq. (2.11), the crossing time is defined as

$$
t_{E}=\frac{r}{\sqrt{\left\langle v^{2}\right\rangle}}=\frac{G m^{5 / 2} N^{5 / 2}}{4 \sqrt{2}|E|^{3 / 2}} .
$$

\footnotetext{
${ }^{1}$ In the following section, we classify all the particles into three species, core, halo, and gas. The typical time scales $t_{R}$ and $t_{\xi}$, respectively, characterize the gas and the core species.

${ }^{2}$ Outside of the above region in SGR model, this form of virial relation would be modified even in the limit of $\xi \rightarrow 0$, as is shown in Appendix A.
}

This is the intrinsic time scale associated with genuine gravity independent of the cutoff $\xi$ and of the system size $R .^{3}$

The above introduced cutoff parameter $\xi$ connects the two limiting cases in the following sense. In the limit $\xi \rightarrow 0$, the SGR model becomes genuine 3D gravity at small scales, while in the limit $\xi \rightarrow R$, the model almost becomes the HMF model. The latter is, because, in this limit, the shape of the potential around $\theta=k \pi(k=0, \pm 1, \ldots)$ becomes almost identical as it does in the HMF model. ${ }^{4}$

For numerical simulations, we need to make all physical variables nondimensional; we use $m, R$, and $t_{R}$ for the unit of mass, distance, and time, respectively. In these units, the physical Hamiltonian (2.1) reads

$$
H_{p}=G m^{2} \frac{N}{R} H,
$$

where

$$
H=\frac{1}{2} \sum_{i=1}^{N} p_{i}^{2}-\sum_{i<j}^{N} \frac{1}{\sqrt{2} N \sqrt{1-\cos \theta_{i j}+\epsilon}} .
$$

The dimensionless momentum $p_{i}$ is given by $p_{i}=d \theta_{i} / d \tau$ and the dimensionless time $\tau$ is introduced as $\tau \equiv t / t_{R}$. The above form of Hamiltonian permits us to use a powerful symplectic integrator [18], with which the total energy is conserved with extremely high accuracy, even beyond thousands of dynamical time. Typical magnitudes of errors for the total Hamiltonian $H(\tau)$ and the total momentum $P(\tau)$ in our simulations up to $\tau \sim 10^{4}$, are $[H(\tau)-H(0)] / H(0)$ $\sim O\left(10^{-5}\right)$ and $P(\tau) / P_{\mathrm{rms}}(\tau) \sim O\left(10^{-8}\right)$, respectively, where $P_{\mathrm{rms}}(\tau) \equiv\left(\sum_{i=1}^{N} p_{i}^{2}\right)^{1 / 2}$.

\section{CLASSIFICATION OF PHASES AND PARTICLES}

We now study the quasiequilibrium state that appears in a transient stationary stage in our SGR system. Though this state is not absolutely stable, it generally appears in SGSs during sufficiently long time before the system finally approaches the equilibrium state characterized by the equipartition of particle energy.

We would like to extract universal properties observed in this transient state; only the transient description is possible and necessary to explain observations.

\section{A. Negative specific heat of SGS}

First, we study the phase diagram of SGR model in the temperature-energy plane. The $T$ - $U$ relation is shown in Fig. 2 and we observe that the region with negative specific heat, i.e., negative slope region, apparently exists. The temperature $T$ and the internal energy per particle $U$ of the present system are defined, respectively, by

\footnotetext{
${ }^{3}$ As we will show in Sec. IV, this is also the time scale for the particles in halo species in SGR model.

${ }^{4}$ The exact HMF model is reproduced in the limit $\xi \rightarrow \infty$.
} 


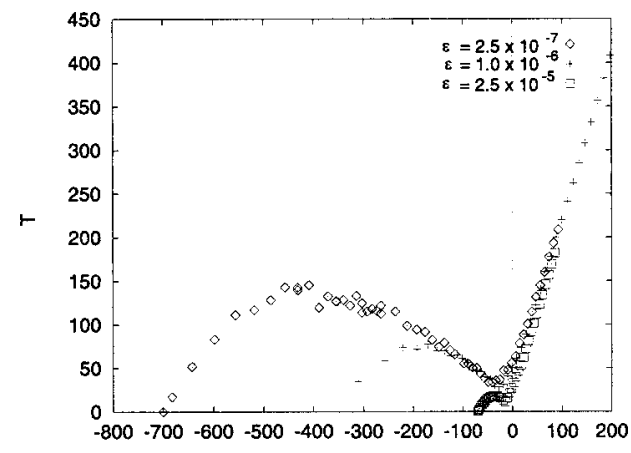

U

FIG. 2. Diagram of temperature $T$ vs energy per particle $U$ for three different cutoffs $\epsilon=2.5 \times 10^{-5}, 1.0 \times 10^{-6}$, and $2.5 \times 10^{-7}$. In each $T-U$ curve, there are two critical energy scales; $U_{c \text {-low }}(\epsilon)$ and $U_{c \text {-high }}(\epsilon)$, between which negative specific heat $\partial U / \partial T<0$ appears.

$$
\begin{gathered}
T \equiv \frac{2\langle K(\tau)\rangle}{N}, \\
U \equiv \frac{H}{N} .
\end{gathered}
$$

The internal energy $U$ is conserved in the present microcanonical system, while the temperature $T$ is defined as the twice of the time-averaged (represented by angle brackets) kinetic energy per particle. The phase diagram in Fig. 2 is time averaged until $\tau=2$ where the system achieves virial equilibrium (see Fig. 3). Throughout this paper, except explicitly mentioned, we fix the total number of particles $N$ $=100$ for simplicity and, therefore, the remaining relevant parameters that characterize the quasiequilibrium state of the system would be $\epsilon$ and $U$.

From Fig. 2, we find two characteristic energy scales where $\partial T / \partial U=0 ; \quad U_{c \text {-low }}(\epsilon)$ at a low-energy side and $U_{c \text {-high }}(\epsilon)$ at a high-energy side. The energy scale $U_{c \text {-high }}(\epsilon)$ corresponds to a mean gravitational binding energy per particle, which is estimated as $\langle 1 /(\sqrt{2} N \sqrt{1-\cos \theta+\epsilon})\rangle[N(N$ $-1) / 2] / N \sim \mathcal{O}(1)$, while $U_{c \text {-low }}(\epsilon)$ strongly depends on the

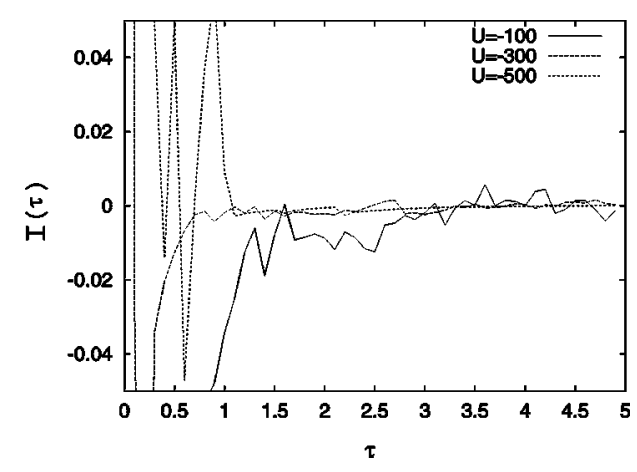

FIG. 3. The time evolution of $I(\tau)$ defined by the left side of Eq. (A5) in the case with $U=-100,-300,-500$, and $\epsilon=2.5 \times 10^{-7}$. In each case, the system achieves virial equilibrium within a few dynamical times $\tau$. cutoff $\epsilon$. This cutoff dependence can be estimated from the condition (2.9) that, in our normalization, becomes

$$
-\frac{1}{4 \sqrt{2 \epsilon}} \ll U \ll-\frac{1}{4} .
$$

Under this condition, the negative specific heat condition in virialized state would be justified. Actually, substitution of three different cutoffs $\epsilon=2.5 \times 10^{-7}, 1.0 \times 10^{-6}$, and 2.5 $\times 10^{-5}$ into Eq. (3.3) yields the lower limits of Eq. (3.3) 353,177 , and 35.3, respectively. Thus the condition (3.3) correctly describes the region of negative specific heat in Fig. 2 with sufficient accuracy. However, the slope of each $T$ - $U$ line in Fig. 2 is less steep than the value -2 which is expected for the virial condition of $3 \mathrm{D}$ gravity. This discrepancy is probably because the energy range satisfying the condition (3.3) is too narrow for the ideal $T$ - $U$ curves according to our choice of the cutoff parameters; even the smallest cutoff we took may not be enough to make the relation converge.

In the range between these two energy scales, i.e., $U_{c \text {-low }}(\epsilon)<U<U_{c \text {-high }}(\epsilon)$, the system has negative specific heat, which suggests the existence of phase transition $[8,15-$ 17]. Actually in the system with negative specific heat, a slight energy injection from outside decreases the system's temperature and induces further energy flow from outside. Then this catastrophic temperature reduction induces rapid cluster formations in the system. As we will see below, such phase transition from the gaseous state to the cluster state actually appears and characteristic structures are realized in this intermediate energy range.

\section{B. Three phases in SGR model}

As is seen in Fig. 2, there are apparently three phases according to the energy per particle $U$; (a) low-energy collapsed phase ( $\mathcal{C}$ phase) $U<U_{c \text {-low }}(\epsilon)$, (b) intermediateenergy phase ( $\mathcal{I}$ phase) $U_{c \text {-low }}(\epsilon)<U<U_{c \text {-high }}(\epsilon)$, and (c) high-energy gaseous phase $\left(\mathcal{G}\right.$ phase) $U_{c \text {-high }}(\epsilon)<U$. The $\mathcal{G}$ phase (c) is stabilized by the infrared cutoff $(\theta \leqslant 2 \pi$, or a largest physical scale $\sim R$ ), without which the particles would escape into spatial infinity. The $\mathcal{C}$ phase (a) is stabilized by the ultraviolet cutoff $\epsilon$, without which the particles would fall into a singularity. The specific heat for these particles (a) and (c) is positive, in accordance with the stability of these phases. On the other hand, the $\mathcal{I}$ phase (b) (the most specific to gravity) has negative specific heat and, therefore, is unstable. The nature of this phase is independent of any artificial cutoffs and, therefore, is thought to represent intrinsic properties of gravity.

The existence of three different phases, as is explained above, distinguishes the SGR model from other models with long-range force. For example, HMF model has only highand low-energy phases, and shows a second-order phase transition between these phases. On the other hand in SGR model, the intermediate phase with negative specific heat exists and is strongly unstable when the system is in contact with a heat bath. As for 1DS model, only a single phase exists because the system has no characteristic energy scale; 
there is no phase transition in this model.

The limit $\epsilon \longrightarrow 0$ in SGR model represents genuine gravity without collision and the range of the intermediate phase increases without bound.

\section{Three species: gas, halo, and core particles}

In each phase, the particles of the system prevail in various energy ranges. For example, in the intermediate phase, some particles evaporate from a cluster and move along the ring almost freely with the time scale $t_{R}$, while some of the others fall into the center of a cluster and oscillate with the time scale $t_{\xi}$. Thus, the overall phase information does not precisely specify the nature of individual particles. In order to obtain much fine information, we define three species in each phase by using the energy of the particles: The energy of the $i$ th particle is given by

$$
E_{i} \equiv \frac{1}{2} p_{i}^{2}-\sum_{j \neq i}^{N} \frac{1}{\sqrt{2} N \sqrt{1-\cos \theta_{i j}+\epsilon}} .
$$

The classification is based on particle energy, that is, (a) core particles for $E_{i}<U_{c \text {-low }}(\epsilon)$, (b) halo particles for $U_{c \text {-low }}(\epsilon)$ $<E_{i}<U_{c \text {-high }}(\epsilon)$, and (c) gas particles for $U_{c \text {-high }}<E_{i} .{ }^{5}$

In the low-temperature phase of SGR, the quasiequilibrium state at very low temperature is highly inhomogeneous and a single cluster is formed. Most particles are condensed in this cluster and the total potential is deep. In the intermediate phase, many particles spread around a cluster. As the temperature increases within this phase, halo particles gradually dominate core particles and eventually there appear gas particles, which evaporate from a cluster and go along the ring. In the high-temperature phase, all particles move almost freely without forming any cluster.

We describe the ratio of particle number in each states ${ }^{6}$ by $\lambda_{\text {core }}, \lambda_{\text {halo }}$, and $\lambda_{\text {gas }}$, with $\lambda_{\text {core }}+\lambda_{\text {halo }}+\lambda_{\text {gas }}=1$. Their evolution is shown in Fig. 4 for the case $\epsilon=2.5 \times 10^{-7}$. From this, we observe those ratios seem to have approached asymptotic values beyond $\tau \approx 1: \quad \lambda_{\text {core }} \approx 0, \lambda_{\text {halo }} \approx 0.2$, and $\lambda_{\text {gas }} \approx 0.8$ for $U=100$ ( $\mathcal{G}$ phase), $\lambda_{\text {core }} \approx 0.41, \lambda_{\text {halo }} \approx 0.23$, and $\lambda_{\text {gas }} \approx 0.36$ for $U=-100$ ( $\mathcal{I}$ phase), $\lambda_{\text {core }} \approx 0.96, \lambda_{\text {halo }}$ $\approx 0.02$, and $\lambda_{\text {gas }} \approx 0.02$ for $U=-500$ ( $\mathcal{C}$ phase). Gas particles dominate in $\mathcal{G}$ phase and core particles dominate in $\mathcal{C}$ phase in number. While in $\mathcal{I}$ phase, all of the three species of particles coexist almost equally. According to our various calculations changing the particle number and initial conditions, this coexistence seems to represent the prominent property of SGS and not the finiteness of particles.

In Fig. 5, the energy dependence of the ratio of each species in $\tau=5$ for $\epsilon=2.5 \times 10^{-7}$ is shown at the center. The energy scale where halo particles exit corresponds to the one where negative specific heat appears ( $\mathcal{I}$ phase). Together

\footnotetext{
${ }^{5}$ Note that even in the $\mathcal{C}$ phase, there exist a few gas particles. We term the core particles and halo particles as a cluster in this paper; they form an apparent single bound state.

${ }^{6} \mathrm{We}$ term a state to represent the situation that a particle belongs to one of the three species.
}
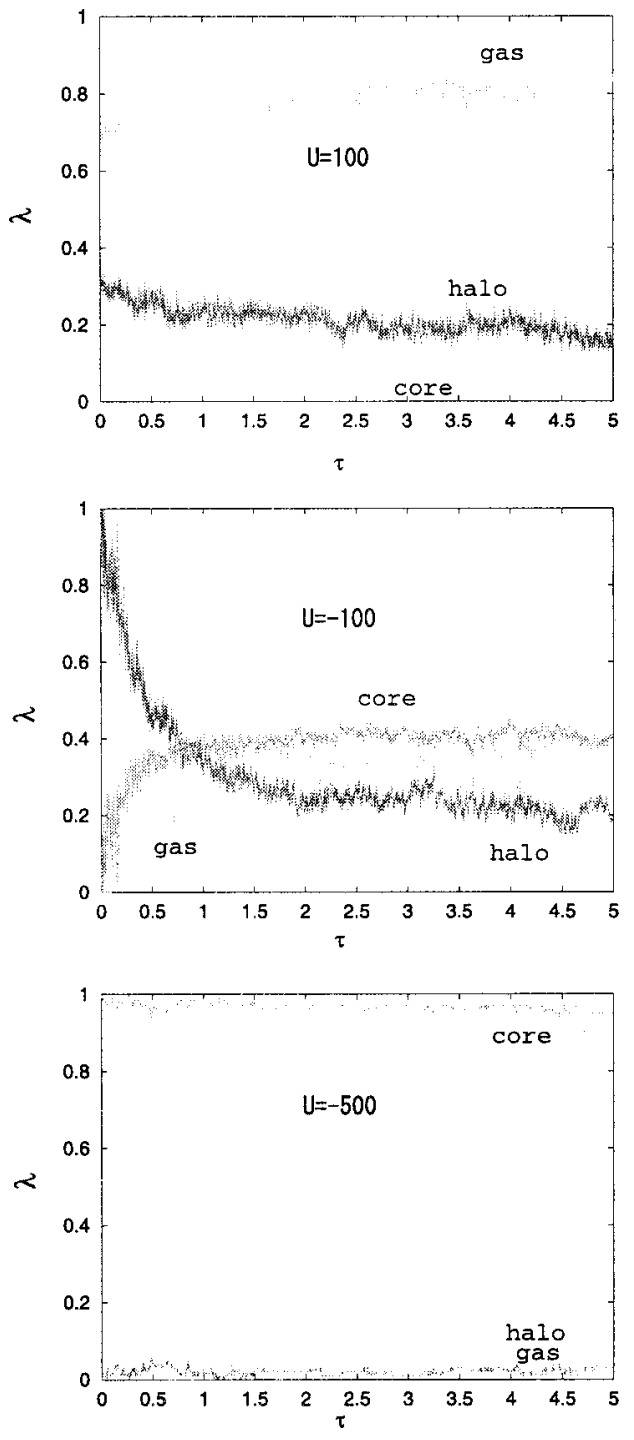

FIG. 4. Time evolution of the percentage of each species: $\lambda_{\text {core }}$, $\lambda_{\text {halo }}$, and $\lambda_{\text {gas }}$ in high-energy (G) phase (top), in intermediateenergy $(\mathcal{I})$ phase (middle), and in low-energy $(\mathcal{C})$ phase (bottom) for $\epsilon=2.5 \times 10^{-7}$. Gas particles dominate in the high-energy phase, and core particles dominate in the low-energy phase. On the other hand, all three species coexist in the intermediate-energy phase.

with it, the relative ratio of time intervals of three states in which each particle stays until $\tau=5$ is shown. As we expect, in both $\mathcal{G}$ and $\mathcal{C}$ phases, most particles stay just in one state for quite a long time. For the $\mathcal{I}$ phase, however, many particles experience at least two states. Some particles wander from one state to another in three states.

\section{PARTICLE MOTIONS}

In this section, we examine individual particle motion and velocity distribution function in each phase.

\section{A. Recurrent motion of halo particles}

In the HMF model at the state near the critical energy, Lévy-type flight and anomalous diffusion of particles have 


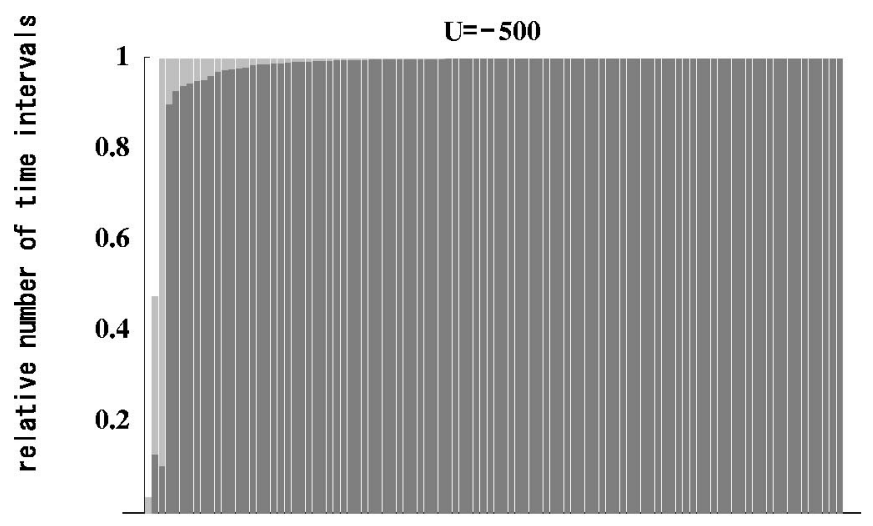

label of particles
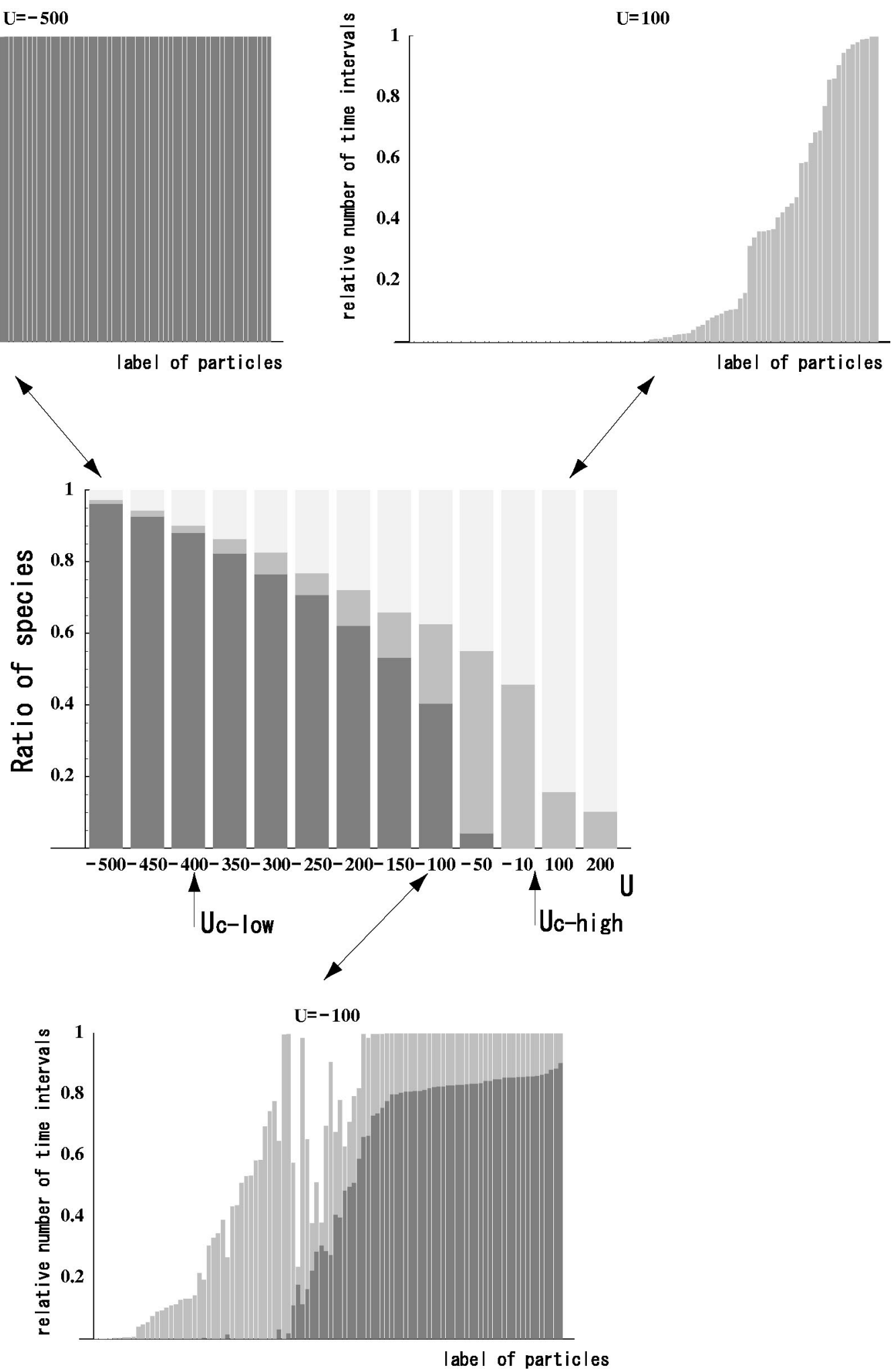

FIG. 5. The middle diagram shows the energy dependence of the ratio of each states at time $\tau=5$ for the cutoff $\epsilon=2.5 \times 10^{-7}$. For three typical cases, $U=100,-100$, and -500 , the ratio of time duration intervals of the three states in which each particle stays until $\tau=5$ is shown in the upper and lower diagrams. In these diagrams, each vertical bar corresponds to a particle, and each ratio of the gas, halo, and core states is represented by the white, gray, and black areas, respectively. In both the $\mathcal{G}$ phase $(U=100)$ and $\mathcal{C}$ phase $(U=-500)$, most particles stay within each state. On the other hand, in the $\mathcal{I}$ phase $(U=-100)$, many particles experience at least two states. Some particles wander in all three states. 
been reported [13]. These peculiar behaviors of particles are apparently caused by the transitions from core particles to gas particles and vice versa. We observe that such peculiar behaviors in HMF model are caused by the periodicity of the configuration space and not by the long-range force itself. In fact, for all Lévy-type flights numerically shown in HMF, the flight distances turn out to be longer than the period $2 \pi$. Therefore, the artificial periodicity in the potential, and not the long-rage nature of the force itself, is thought to have caused the Lévy-type flight in HMF model. In our SGR model, we do have the possibility to observe the same Lévytype flight motions since particles in SGR also move along a closed ring. Since we would like to extract the intrinsic property of the long-range nature of the gravity itself, we pay attention to the recurrent motion of halo particles and disregard the round-trip motion along the entire ring.

First, we choose the parameters as $\xi \approx 7.1 \times 10^{-4} R$ and $U=-100$ for which the system is in the intermediate-energy phase. The particle motion is shown in Fig. 6(a), in which core particles form a firm cluster and they oscillate around the center of the cluster with the time scale $t_{\xi}$ and halo particles go in and out of the core region without any typical time scale and amplitude. Zoomed in ten times [Fig. 6(b)], and even in hundred-times [Fig. 6(c)], the particle motion is always similar recurrent movements. This repetition of the similar recurrent pattern suggests a self-similar structure of the system.

Note that the recurrent motion of halo particles is confined within the range $[0,2 \pi]$ and particles never experience a round trip along the ring, quite contrary to the HMF model. Moreover, this recurrent motion of particles is quite robust and is observed in any region of the intermediate energy phase. This robustness is a remarkable contrast to the HMF model in which such motion is observed only at the critical point in the phase diagram. ${ }^{7}$

In order to analyze this behavior more quantitatively, we examine the frequency distribution of the recurrent time $\tau_{\text {rec }} \equiv \tau_{\text {in }}-\tau_{\text {out }}$, that is, the time period from the moment $\tau_{\text {out }}$ when a particle leaves the barycenter of the core to the moment $\tau_{\text {in }}$ when it first returns to the barycenter again. Here we have defined the location of the barycenter of the core as

$$
\theta_{b c} \equiv \sum_{i=1}^{N_{c}} \theta_{i} / N_{c}
$$

where $\theta_{i}$ is the location of $i$ th particle in the core and $N_{c}$ is the total number of core particles at each moment.

In Fig. 7, we depicted the frequency distribution of the recurrent time $\tau_{\text {rec }}$ for core particles and halo particles separately. For core particles, as we expected, we find almost Gaussian distribution around the center $\tau_{\xi}$ [Fig. 7(a)]. On the other hand for halo particles, although a peak is found around $\tau_{E}\left(\equiv t_{E} / t_{R}\right)$, the distribution shows that a long tail spreads widely characterized by the power law $f\left(\tau_{\text {rec }}\right)$ $\sim \tau_{\text {rec }}^{-p}$ with $p \approx 2.0$ [Fig. 7(b)]. We have checked that this

\footnotetext{
${ }^{7}$ The smaller the cutoff $\epsilon$, the larger is the range of the total energy per particle $U$ where the recurrent motion appears.
}
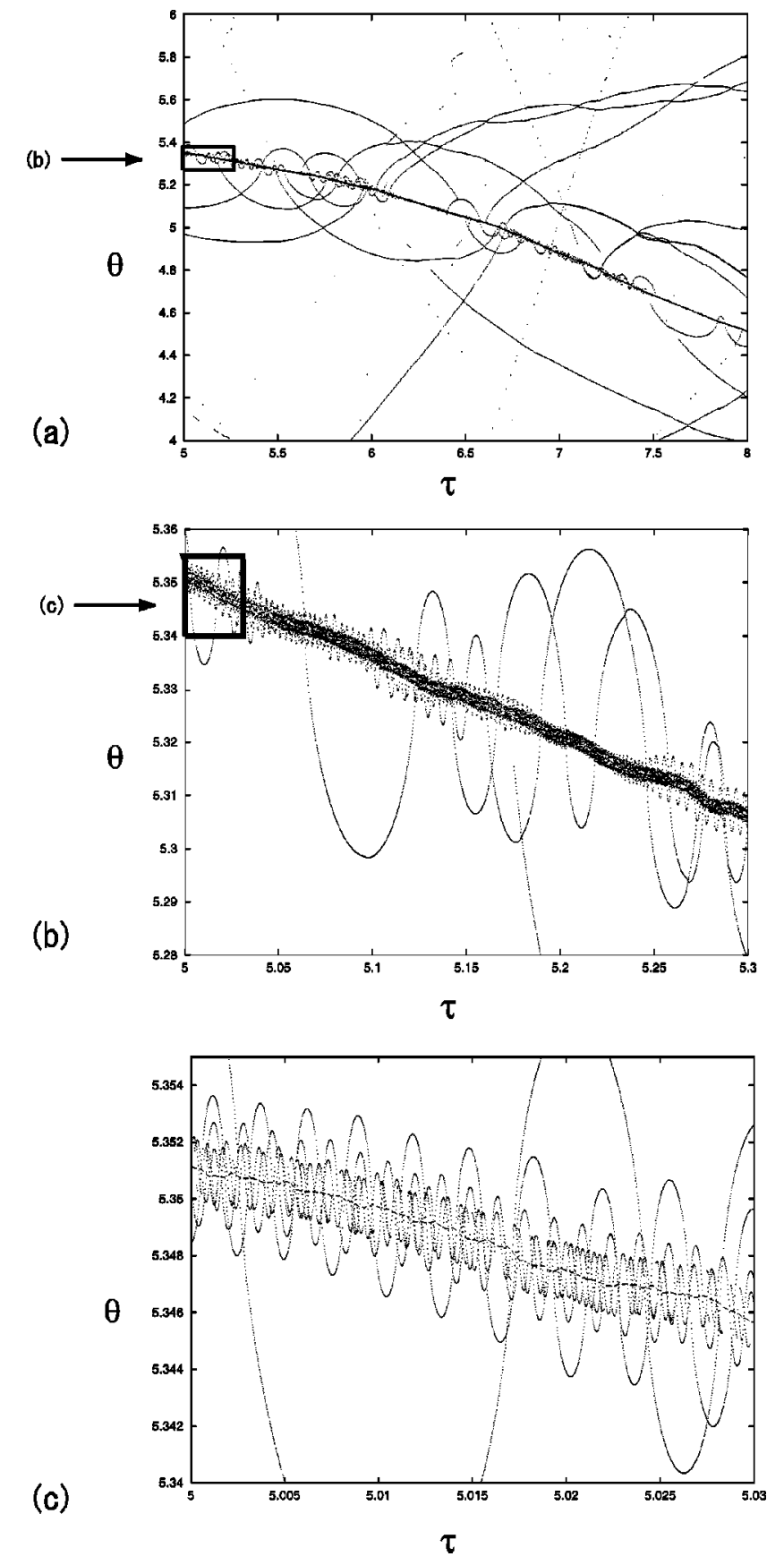

FIG. 6. Trajectories of halo particles for the time interval (a) $\tau=5-8$, (b) $\tau=5-5.3$, and (c) $\tau=5-5.03$. Ten-times zooming up the marked square region in (a) yields (b). Further ten-times zooming up the marked square region in (b) yields (c). The recursion profiles are similar despite the scale difference.

power law range $-3<\log _{10} \tau_{\text {rec }}<-1.5$ is consistent with the eye-fitted region of self-similar motion in Fig. 6. We will see that the above difference of frequency distribution for core and halo particles leads to the difference in relaxation time of them in Sec. V.

We have also examined the energy dependence of the above power $p$. The result is given in Fig. 7(b) showing that the value of $p(\approx 2.0)$ is almost independent of the choice of the energy $U$ throughout the intermediate-energy phase. 


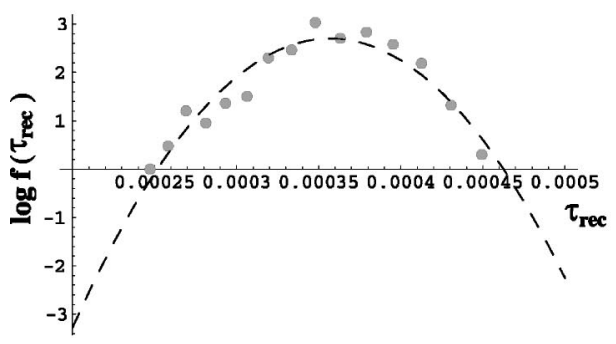

(a)

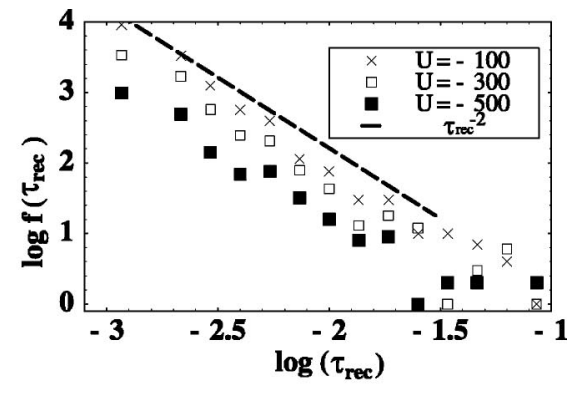

(b)
FIG. 7. A linear-log plot with common logarithm of frequency distribution $f\left(\tau_{\text {rec }}\right)$ for core particles and a logarithmic plot of frequency distribution $f\left(\tau_{\text {rec }}\right)$ for halo particles in several values of $U$. Each plot in (b) shows the same slope within the range $10^{-3.0}<\tau_{\text {rec }}<10^{-1.5}$. The broken line represents a line with slope -2.0 .

\section{B. Velocity distribution of particles}

As is shown in Appendix A, the time averaged kinetic energy $\langle K\rangle$ is, in general, expressed as the sum of $N^{2}$ independent stochastic variables. However, in the case of core particles, which are located within a few cutoff scales, the quantity $\langle K\rangle$ turns out to be expressed as the sum of $N$ independent stochastic variables (see Appendix B). In this section, we show that the $N$ dependence of $\langle K\rangle$ characterizes the velocity distribution of particles.

In Fig. 8, we show the velocity distributions of core and halo particles in three cases; (a) $\xi \approx 5 \times 10^{-4} R, \quad U=$ $-100 \quad$ (b) $\xi \approx 5 \times 10^{-4} R, \quad U=-500, \quad$ and $\quad(\mathrm{c}) \quad \xi \approx 5$ $\times 10^{-2} R, \quad U=-0.65$. We have superposed the velocity data at $\tau=1,2,3,4$, and 5 , with ten different random initial conditions fixing the total energy. Thus the size of the whole data we used is $5 \times 10^{3}$ for each velocity distribution function. The distribution of core particles is well fitted by the Gaussian distribution

$$
P(v)=\frac{1}{\sqrt{2 \pi} \sigma} e^{-v^{2} / 2 \sigma^{2}},
$$

with the dispersion $\left\langle v_{\text {core }}^{2}\right\rangle^{1 / 2} \equiv \sigma=7.29(\mathrm{a}), 10.9(\mathrm{~b})$, and $0.51(\mathrm{c})$.
On the other hand, the distribution of halo particles in (a) is manifestly non-Gaussian, although that of the same particles in the case (c) is Gaussian. This distribution is at least unchanged until $\tau=200$.

What is the origin of the above non-Gaussian distribution for halo particles in the intermediate-energy phase in case (a)? Here we analyze this issue from the viewpoint of the generalized central-limit theorem. It is well known that the limit distribution for sums of independent random variables is the Gaussian distribution provided that the dispersion is finite (the central-limit theorem). However, it is less well known that the limit distribution for sums of independent random variables is the stable distribution in general cases, including those where the dispersion is divergent. This stable distribution is defined to satisfy the relation

$$
\sum_{i=1}^{N} x_{i} \stackrel{d}{=} N^{1 / \alpha} x
$$

where $\underline{\underline{d}}$ means that the distributions of both sides are equal to each other. The parameter $\alpha$ classifies the stable distributions and must satisfy $0<\alpha \leqslant 2$ for the normalizability and the positivity of the probability distribution function. In the above definition, $x_{i}$ and $x$ are the probabilistic variables obeying the same distribution. Let us apply this to the veloc- (a)

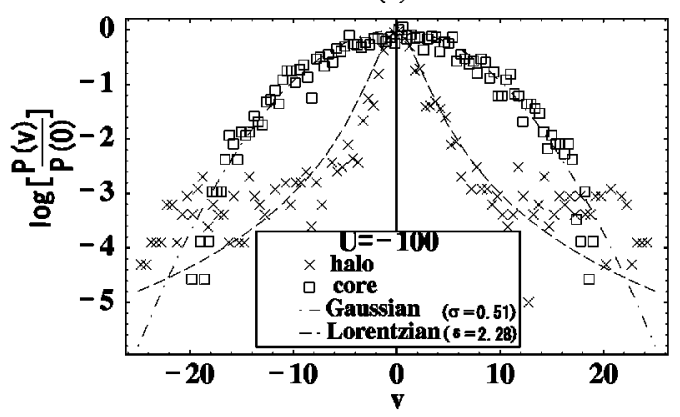

(b)

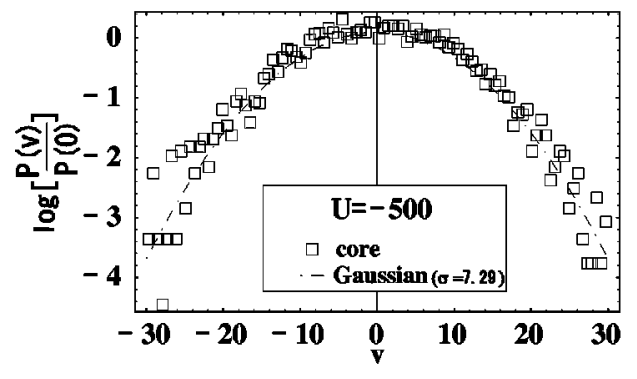

(c)

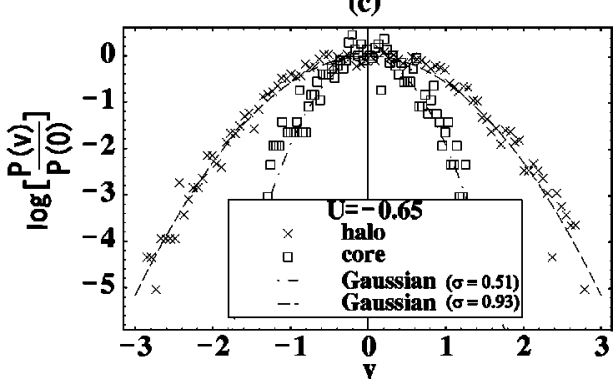

FIG. 8. The linear-log plot with common logarithm of velocity distributions of particles for the three cases: (a) $\epsilon=2.5$ $\times 10^{-7}, \quad U=-100 \quad(\mathcal{I}$ phase $)$, (b) $\epsilon=2.5 \times 10^{-7}, \quad U=-500$ (C phase), and (c) $\epsilon=2.5$ $\times 10^{-3}, \quad U=-0.65$ ( $\mathcal{I}$ phase $)$. In all cases, velocities of core particles are well fitted by the Gaussian distribution with dispersion $\sigma$ $=0.51(\mathrm{a}), 7.29(\mathrm{~b})$, and 0.51 (c), respectively. Also in the large cutoff case (c), velocities of halo particles are well fitted by the Gaussian distribution $\sigma=0.93$. On the other hand, in the small cutoff case (a), velocities of halo particles are well fitted by the Lorentzian distribution $(\alpha=1)$ with $s$ $=2.2$. 
ity distribution of our model. We square both sides of the above equation, and obtain the nonextensive property

$$
\left\langle v^{2}\right\rangle_{N \text {-particle }}=N^{2 / \alpha-1}\left\langle v^{2}\right\rangle_{\text {one-particle }}
$$

and the method of characteristic function yields the explicit form of the distribution function [19]

$$
P(v)=\frac{1}{2 \pi} \int_{-\infty}^{\infty} d y \exp \left[-i v y-s|y|^{\alpha}\right] .
$$

Note that this stable distribution includes the Gaussian distribution (4.2) as a special case $\alpha=2$, where the kinetic energy becomes extensive, and the dispersion $\sigma=\sqrt{2 s}$ is finite. The parameter $s$ is thought to be a generalized temperature.

Let us first consider the velocity distributions of core particles. We observe, from our numerical calculations, that all core particles oscillate within the narrow region of the cutoff size $\xi$, and the gravitational two-body interaction is dominated by the artificial potential force. Therefore, the averaged kinetic energy is described as the sum of $N$ independent statistical elements (Appendix B). This leads to the normal extensivity for the velocity distributions of core particles and, therefore, we expect the Gaussian distribution. This is consistent with the results in Fig. 8.

On the other hand, for halo particles that interact through genuine gravity, the kinetic energy behaves as $\left\langle K_{p}\right\rangle \sim N^{2}$ for fixed $R$ as is shown in Appendix A. Thus from Eq. (4.4) we find the index $\alpha=1$ for the physical velocity distribution of SGR. In this case with $\alpha=1$, the distribution (4.5) becomes the Lorentz form

$$
P(v)=\frac{1}{\pi} \frac{s}{v^{2}+s^{2}}
$$

This is also consistent with the results in Fig. 8. The essence of the appearance of this non-Gaussian distribution is the nonextensivity of the energy for SGS; this is the intrinsic property of gravity.

Then what is the origin of the Gaussian distribution of halo particles in case (c)? As we show in the following section, this is mainly because the relaxation times of core and halo particles are close to each other. In the following section, we will discuss the relation between the profile of velocity distribution and the relaxation process.

\section{RELAXATION}

One of the most important issues in statistical physics of the $N$-particle system is the relaxation process. In order to study the relaxation process of our model, we choose several different initial conditions for the same values of $\epsilon$ and $U$. Here we have examined two parameter sets, i.e., (i) $\epsilon=2.5$ $\times 10^{-3}, U=-0.65$ and (ii) $\epsilon=2.5 \times 10^{-7}, U=-100$, for both of which negative specific heat appears.

As we discussed in Sec. II, there are three dynamical time scales: $t_{R}, t_{\xi}$, and $t_{E}$. In the units of our normalization, the ratios of these time scales are

$$
\begin{gathered}
t_{E} / t_{R}=\frac{1}{4 \sqrt{2}}|U|^{-3 / 2}, \\
t_{\xi} / t_{R}=(2 \epsilon)^{3 / 4} .
\end{gathered}
$$

For example, if we choose $U=-100$ and $\epsilon=2.5 \times 10^{-7}$, then $t_{E} / t_{R} \approx 1.8 \times 10^{-4}$ and therefore the three time scales are separated as

$$
t_{\xi}<t_{E} \ll t_{R}
$$

Then the contribution of gas particles to the dynamics of halo or core particles is negligible during a few $t_{R}$.

The parameter set (i) does not meet the condition (3.3) and $t_{E} / t_{R} \approx 0.34 ; \quad t_{E}$ is the same order as $t_{R}$. On the other hand, the parameter set (ii) meets Eq. (3.3) and $t_{E} / t_{R} \approx 1.8$ $\times 10^{-4} ; \quad t_{E}$ is much shorter than $t_{R}$. This means that gas species play an important role in relaxation in case (i), while they do not do so in case (ii). In order to examine the relaxation process for these two cases, it is convenient to normalize the time with the unit $t_{E}$, since the degree of relaxation of $3 \mathrm{D}$ gravitational system is conventionally measured with the dynamical time. So here we introduce the dimensionless time $\tau_{E} \equiv t / t_{E}$.

As for initial conditions, we locate several clusters of the same size with the same interval. The number of clusters is chosen as 5, 10, 20, 25, and 50 for model (i) and 5, 10, 20, and 25 for model (ii). The initial velocities of particles are set randomly but the total energy is fixed: $U=-0.65$ for (i) and $U=-100$ for (ii).

In order to study the Ergode property of the system, we examine the mixing property of the particles in the three states (core, halo, and gas). In other words, we examine the degree of isolation of each states. We directly measure the extent to which each particle experiences these three states. First, we pick up discrete times $\left(\tau_{E 1}, \tau_{E 2}, \ldots, \tau_{E i}, \ldots\right)$ with the equal interval $\Delta \tau_{E}$; at each time, each particle stays at one of the three states. Then we count how many times the $k$ th particle stays at each states before the time $\tau_{E i}$. Finally, after normalization, we obtain the relative frequencies of three states $\lambda_{\text {core }}^{k}\left(\tau_{E i}\right), \lambda_{\text {halo }}^{k}\left(\tau_{E i}\right)$, and $\lambda_{\text {gas }}^{k}\left(\tau_{E i}\right)$ (with $\left.\lambda_{\text {core }}^{k}\left(\tau_{E i}\right)+\lambda_{\text {halo }}^{k}\left(\tau_{E i}\right)+\lambda_{\text {gas }}^{k}\left(\tau_{E i}\right)=1\right)$ for the $k$ th particle.

At the first time $\tau_{E 1}$, each particle definitely stays at one of the three states; relative frequencies of stay $\lambda_{\text {core }}^{k}\left(\tau_{E 1}\right), \lambda_{\text {halo }}^{k}\left(\tau_{E 1}\right)$, and $\lambda_{\text {gas }}^{k}\left(\tau_{E 1}\right)$ are either 1 or 0 . In the next time $\tau_{E 2}$, some particles may change the state; then $\lambda_{\text {core }}^{k}\left(\tau_{E 2}\right), \lambda_{\text {halo }}^{k}\left(\tau_{E 2}\right)$ and $\lambda_{\text {gas }}^{k}\left(\tau_{E 2}\right)$ for these particles are $1 / 2$. In this way, $\lambda_{\text {core }}^{k}\left(\tau_{E i}\right), \lambda_{\text {halo }}^{k}\left(\tau_{E i}\right)$ and $\lambda_{\text {gas }}^{k}\left(\tau_{E i}\right)$ will evolve in time $\tau_{E i}$. Various distributions of $\left\{\lambda_{\text {core }}^{k}\left(\tau_{E i}\right)\right\}_{k=1}^{N}$ for all the particles $k=1,2, \ldots, N$ will define the distribution function $N\left(\lambda_{\text {gas }}, \tau_{E}\right)$ for values $\lambda_{\text {gas }}$ at time $\tau_{E i}$ by simply counting the number of the particles that take the value $\lambda_{\text {core }}$ at time $\tau_{E}$. Similarly distribution functions for other states can also be defined: $N\left(\lambda_{\text {halo }}, \tau_{E}\right)$ and $N\left(\lambda_{\text {core }}, \tau_{E}\right)$. As the system is thermally relaxed and the equipartition of energy is attained, $\lambda_{\text {core }}^{k}\left(\tau_{E}\right), \lambda_{\text {halo }}^{k}\left(\tau_{E}\right)$, and $\lambda_{\text {gas }}^{k}\left(\tau_{E}\right)$ will converge to the same value $\lambda_{\text {core }}^{*}, \lambda_{\text {halo }}^{*}$, and $\lambda_{\text {gas }}^{*}$ independent of the particle label $k$. Then $N\left(\lambda_{\text {gas }}, \tau_{E}\right), \quad N\left(\lambda_{\text {halo }}, \tau_{E}\right)$, and 

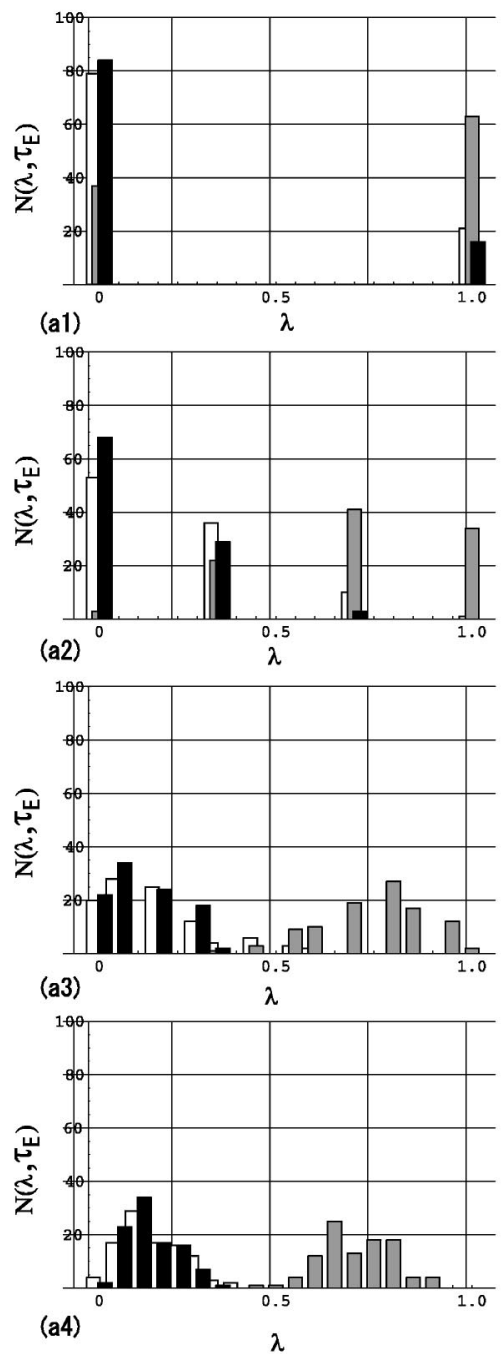
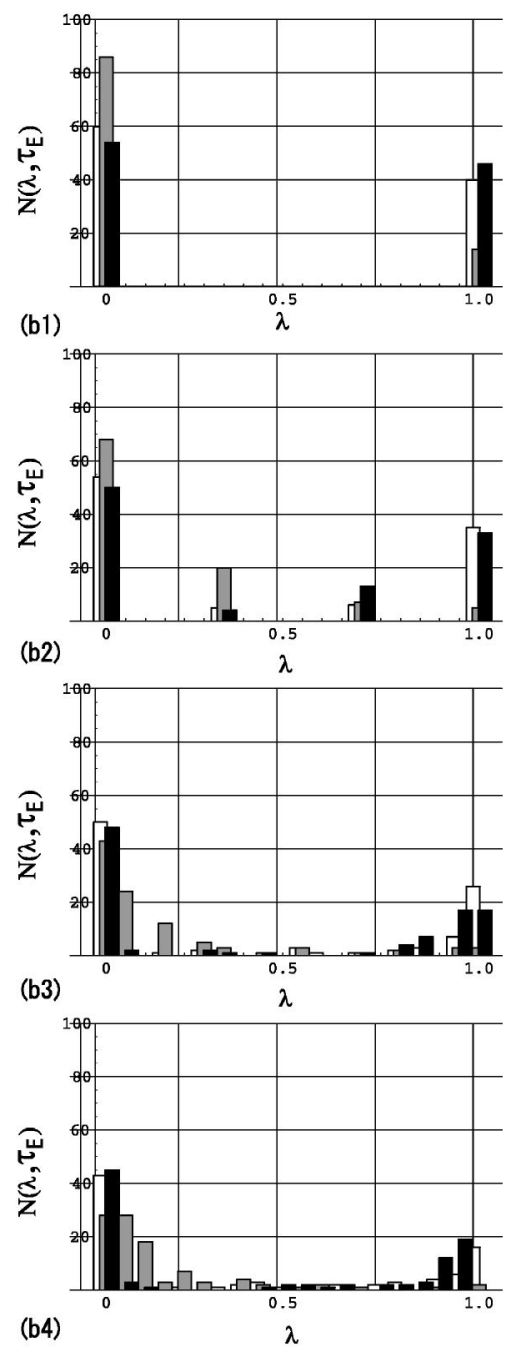

FIG. 9. Time variation of $N\left(\lambda, \tau_{E}\right)$, where the bars in white, gray and black colors show $N\left(\lambda_{\text {gas }}, \tau_{E}\right), N\left(\lambda_{\text {halo }}, \tau_{E}\right)$, and $N\left(\lambda_{\text {core }}, \tau_{E}\right)$, respectively. The left and right columns, respectively, represent the case of large UV cutoff (a) $\epsilon=2.5 \times 10^{-3}$ and $U=-0.65$ and the case of small UV cutoff (b) $\epsilon=2.5 \times 10^{-7}$ and $U=$ -100 , where $\tau_{E 1}=6.5 \times 10^{4}, \Delta \tau_{E}=2.0 \times 10^{3}$ for (a), and $\tau_{E 1}=1.1 \times 10^{5}, \Delta \tau_{E}=3.8 \times 10^{3}$ for (b). In both columns, time flows from top to bottom: $(a 1, b 1) \tau_{E_{1}},(a 2, b 2) \tau_{E_{3}},(a 3, b 3) \tau_{E_{12}},(a 4, b 4) \tau_{E_{34}}$, respectively.
$N\left(\lambda_{\text {core }}, \tau_{E}\right)$ would have sharp peaks at $\lambda_{\text {core }}^{*}, \lambda_{\text {halo }}^{*}$, and $\lambda_{\text {gas }}^{*}$ in the thermal equilibrium state. Thus the development of the peaks in the distribution functions $N\left(\lambda_{\text {gas }}, \tau_{E}\right), N\left(\lambda_{\text {halo }}, \tau_{E}\right)$, and $N\left(\lambda_{\text {core }}, \tau_{E}\right)$ can be a good measure of the degree of thermal equilibrium.

In the large UV-cutoff case (i), each distribution $N\left(\lambda_{\text {gas }}, \tau_{E}\right), N\left(\lambda_{\text {halo }}, \tau_{E}\right)$, and $N\left(\lambda_{\text {core }}, \tau_{E}\right)$ starting from $\tau_{E 1}=6.5 \times 10^{4}$ seems to develop a single peak in between 0 and 1 at least by the time $\tau_{E} \approx 10^{5}$ [Fig. 9(b)], and the variance around the peak seems to reduce in time [Fig. 9(c)]. This means that most particles experience all of the three states at least by $\tau_{E} \approx 2.0 \times 10^{5}$; thermal relaxation proceeds in the time scale $\tau_{E} \sim 10^{5}$. In contrast, in the small UV-cutoff case (ii), each distribution $N\left(\lambda_{\text {gas }}, \tau_{E}\right), N\left(\lambda_{\text {halo }}, \tau_{E}\right)$, and $N\left(\lambda_{\text {core }}, \tau_{E}\right)$ starting from $\tau_{E 1}=1.1 \times 10^{5}$ seems to develop peaks at the edge of the domain 1 and/or 0 , even after the time duration, $\tau_{E} \approx 3.8 \times 10^{5}$ [Fig. 9(e)]. This means that most particles stay in each single state even in the time scale $\tau_{E} \sim 10^{5}$; the system does not approach thermal relaxation at all in this time scale.

By combining these results, the Gaussian velocity distribution realized in case (i) in Fig. 8 seems to reflect the achieved thermal relaxation of the system. On the other hand, the Lorentzian velocity distribution realized in case (ii) in Fig. 8 seems to reflect some quasiequilibrium intermediate stage before the thermal relaxation of the system. Latter peculiar properties in velocity distributions may make 3D gravitational system quite different from those in the system with short-range interactions or the system with positive specific heat. If we set $\epsilon$ larger, then the encounter of halo particles with normal particles (gas and core particles) becomes more frequent, and the halo particles cannot be isolated from the normal particles, resulting in trivial thermodynamical equilibrium of the whole system as in case (i).

\section{SCALING PROPERTIES OF THE HALO}

In Sec. IV, we have found that the velocity distribution of halo particles is non-Gaussian with anomalous $v^{-2}$ tail and each halo particle shows intermittent recursive motion around the core without a definite time scale. These scalefree properties of the halo particles turn out to appear in the spatial distribution profile of them itself. In this section, we study the scaling property of the spatial distribution with the box-counting method [20].

For this purpose, we use the box counting method: We divide the entire configuration space $2 \pi R$ into segments with equal size $\ell$ and count the number $N(\ell)$ of the segment 
that contains at least one halo particle. Then we define the quantity $-\partial\left(\log _{10} N(\ell)\right) / \partial\left(\log _{10} \ell\right)$, which turns out to be the scaling exponent, provided that the quantity is almost independent of the scale $\ell$. In order to increase the statistical significance, we used multiple particle-distribution data at different times for each run. Moreover, we made many runs of calculations with different initial conditions for the fixed total energy $U$. We have applied the box-counting method to each data and then all the individual results are superposed. When we extract the distribution data, we have chosen the time interval $\left[2000 t_{\xi}, 5000 t_{\xi}\right]$, in which the particle ratios $\lambda_{\text {core }}, \lambda_{\text {halo }}$, and $\lambda_{\text {gas }}$ almost reach the relaxed constant values. Thus we calculate the following averaged quantity:

$$
D(\ell) \equiv-\left\langle\frac{\partial\left(\log _{10} N(\ell)\right)}{\partial\left(\log _{10} \ell\right)}\right\rangle_{\mathrm{ens}},
$$

over all the data we thus prepared. If any scaling property exists in the particle distribution, $D(\ell)$ would become constant for a finite range of $\ell_{1} \leqslant \ell \leqslant \ell_{2}$. As for the bounds $\ell_{1}$ and $\ell_{2}$, we have technical restrictions originating from our numerical calculation method. The averaged distance between gas particles is estimated as

$$
l_{\text {gas }}(\epsilon) \sim \frac{2 \pi R}{N \lambda_{\text {gas }}} .
$$

If the box size $\ell$ is larger than $l_{\text {gas }}(\epsilon)$ we expect $D \approx 1.0$, because almost all the boxes contain at least one particle. On the other hand, if $\ell$ is smaller than the cutoff distance $\xi$, the genuine property of gravity is lost. Therefore, scaling property in particle distribution is relevant only within the range $\xi \leqslant \ell \leqslant \ell$ gas. Actually in our calculation, for $\epsilon=2.5 \times 10^{-7}$ and $U=-100$, we can see the typical behavior of $N(\ell)$ as a function of $\ell$ in Fig. 10. In this plot, we can see scaling behavior $N(\ell) \sim \ell^{-d}$ with the small exponent in the range $10^{-3} R<\ell<10^{-1} R$, which is well inside the relevant region. This scaling seems to originate from halo particles, because they not only dominate in the above scaling range but also show non-Gaussian velocity distributions and self-similar recursion jumps.

However, the scaling property of the box-counting method does not always conclude the existence of the fractal structure in the particle distributions. This is because the box-counting method itself cannot distinguish the two possibilities; (a) genuine fractal structure and (b) the power-law tail of the particle distributions around the core center.

In order to distinguish the above possibilities, we compare two different superposition methods as follows: (a) We simply superpose all the data with the bare coordinate $\theta$ and (b) we superpose all the data with the coordinate adjusting so that the mass center of each data comes to the same position, i.e., we introduce a new coordinate $\bar{\theta}$ as $\bar{\theta} \equiv \theta-\theta_{b c}$ for each data with the mass center $\theta_{b c}$.

As a result, the scaling property of the particle distributions, which was observed in the original method, disappears completely in case (a). On the other hand in case (b) we observe the same scaling property as was observed in the original method. This result suggests that the observed scal-

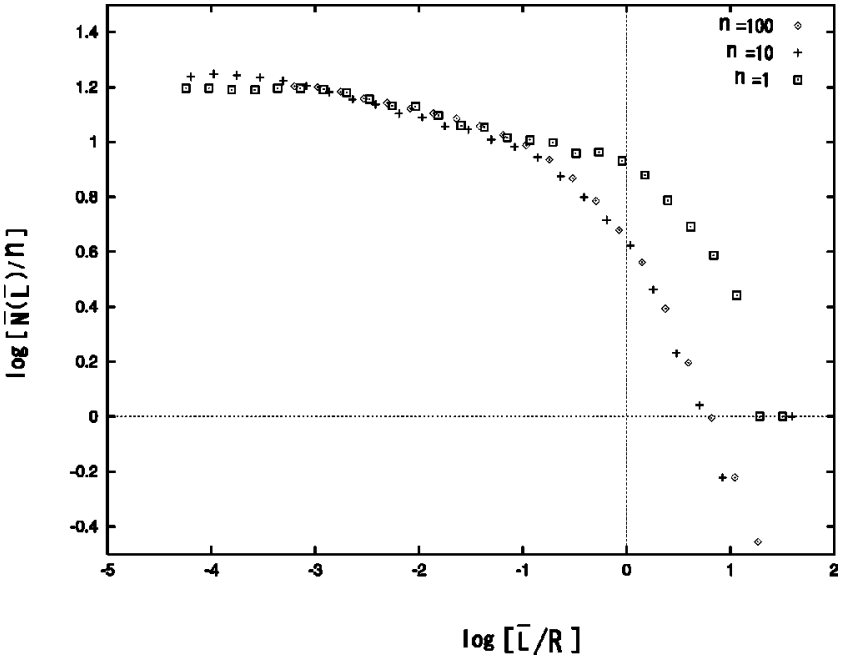

FIG. 10. Log-log plot with common logarithm for the box scale $\bar{L}$ vs the number of the box $\bar{N}$ occupied by at least one halo particle. We superposed the snapshot data of $n$ different times. The cases with $n=1,10\left(\tau_{0}=2.0, \Delta \tau=0.3\right)$, and $100\left(\tau_{0}=2.0, \Delta \tau=0.03\right)$ with the cutoff $\epsilon=2.5 \times 10^{-7}\left(\xi \approx 7.1 \times 10^{-4} R\right)$ are plotted. The plots of these three cases coincide with each other in the range $\xi$ $<\bar{L}<10^{-1} R$. The scaling exponent $D(\bar{L})$ derived from the slope in this range is 0.1 .

ing property in particle distribution is not the genuine fractal property of the system, but simply due to the power-law distribution of particles around the mass center. We have confirmed this with our further analysis on the direct observation of the power-law particle distributions around the mass center.

The energy and cutoff dependence on the scaling exponent $D$ is shown in Fig. 11. Here we show the scaling exponent of halo particles in the region of negative specific heat. We find that the scaling exponent $D$ is almost constant in this region. Moreover, smaller cutoff $\epsilon$ corresponds to the smaller dimension $D$. On the other hand in the limit of $\epsilon$ $\rightarrow 1$, the scaling property cannot be observed.

In the limit of real gravitational interaction $(\epsilon \rightarrow 0)$, the region of negative specific heat extends in energy range and

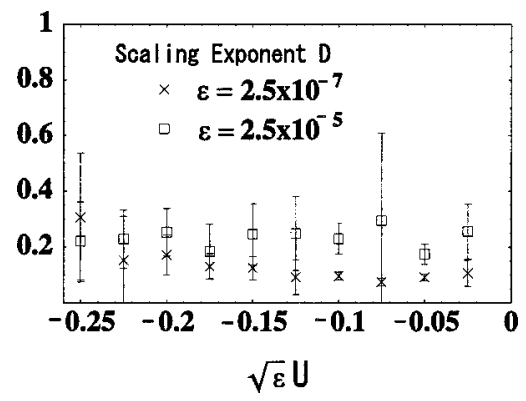

FIG. 11. Scaling exponent $D$ vs energy per particle $U$. Most of the energy region shown in the figure corresponds to the $\mathcal{I}$ phase. For convenience, we use $\sqrt{\epsilon} U$ as a horizontal axis. The case of $\epsilon$ $=2.5 \times 10^{-5}$ (squares with error bars) and the case of $\epsilon=2.5$ $\times 10^{-7}$ (crosses with error bars) are plotted. It seems that the dimension $D$ does not depend on $U$. Since the number of halo particles is small in low energy, the error becomes large there. 
so does the scaling region. This constant property of the exponent $D$ is likely to be independent of the temperature or the energy of the system.

\section{CONCLUSION}

In this paper we have studied the self-gravitating ring (SGR) model that is a one-dimensional (1D) model of a selfgravitating system. Although the system is $1 \mathrm{D}$, the particles constrained on a circular ring mutually interact with the 3D full gravitational force. We found that an interesting phase with the negative specific heat appears at the intermediateenergy scale, which reflects the virial condition of the 3D gravitating system. Classifying the particles in each phase into three species (core, halo, and gas specie), we examined dynamical properties of this phase. Using SGR model, we further examined characteristic properties of relaxation, velocity distributions, and density profiles in each phase.

The cutoff parameter $\xi$ introduced in SGR model controls the singular properties of the system at short distances. By changing this parameter, we could systematically study the effect of the short-distance singularity of the force upon the statistical properties of the system.

When the cutoff parameter is of order one $(\xi \sim R)$, the system resembles the Hamilton mean-field model (HMF). In fact, the velocity distribution becomes Gaussian at $t$ $\sim O\left(10^{3}\right) t_{R}$. This is because the two time scales $t_{E}$ and $t_{R}$ become of the same order and, therefore, halo particles frequently encounter and interact with gas particles. This strong interaction induces frequent exchange of particles between these two species. Thus the energy diffuses effectively and complete thermodynamical equilibrium of the entire system is established. Especially the temperature is the same for all the particle species. In other words, the negative specific heat region (halo particles) does exist, but the strong interaction with gas and core particles (positive specific heat) quickly dissolves the negative specific heat region.

In much more smaller cutoff case with $\xi \approx 7.1$ $\times 10^{-2} R\left(\epsilon=2.5 \times 10^{-3}\right)$, the velocity distribution becomes almost Gaussian after the virialization, although the temperature of each species is different from each other. This temperature difference, as well as the required time for establishing the equilibrium, is increased for smaller value of the cutoff $\xi$.

On the other hand, as $\xi \rightarrow 0$, the difference of the two time scales increases $t_{R} \gg t_{\xi}$, and the energy region of the negative specific heat extends wider. Therefore, we can expect that halo particles become almost isolated from the gas particles. This isolation of unstable halo particles from other normal species enables the halo species to last sufficiently long. In the case of $\xi \approx 7.1 \times 10^{-4} R\left(\epsilon=2.5 \times 10^{-7}\right)$, the initial condition dependence of halo particles survives even after $5 \times 10^{4} t_{R}$ in our simulation, which prevents the full system from reaching thermal equilibrium. These results resemble the case of core particles with $\xi \approx 7.1 \times 10^{-2} R$. However, the remarkable difference between the two cases is that the velocity distribution of halo particles in $\xi \approx 7.1$ $\times 10^{-4} R$ is not Gaussian but Lorentzian distribution, while that of core particles in $\xi \approx 7.1 \times 10^{-4} R$ is Gaussian. This property of halo particles qualitatively reflects genuine $3 \mathrm{D}$ gravitational interaction and is the main difference from the HMF models or 1DS models We have also found the scaling structure of halo particles in the intermediate-energy phase. When we reduce the cutoff $\xi \rightarrow 0$, the scaling region as well as the energy range of negative specific heat is extended wider, although the value of exponent depends on the cutoff and reduces with $\xi$.

In 1DS model, the system reaches thermodynamical equilibrium at least at $t \sim O\left(10^{7}\right) t_{E}$, where $t_{E}$ $\equiv(1 / 4 \pi G m N) \sqrt{4 E / m N}$ is the crossing time of 1DS model [10]. It is also shown that the system reaches the collisionless mixing phase in much shorter time interval. Our simulation with $\xi \approx 7.1 \times 10^{-4} R$ shows non-Gaussian velocity and scaling property in such a short time interval $4 \times 10^{3} t_{E} \sim 2$ $\times 10^{5} t_{E}$ for $U=-100$. So the non-Gaussian velocity distribution might reflect the character in collisionless mixing phase that appears in 1DS simulation [10]. However, it might also be true that the relaxation process in SGR is quite different from that in 1DS, where no time scale separation between core and halo particles exists. So the exotic character of halo particles might be intrinsic in SGR or the system with real 3D gravitational interactions because of the time scale separation.

In summary, SGR model is characterized (i) by the phase separation and the particle state separation and (ii) by similar recurrent motion and non-Gaussian velocity distribution of halo particles. Both these characters may play a key role in explaining the observational properties in real 3D selfgravitating objects. For example, the recent observations with Hubble space telescope support the existence of supermassive black holes or cusps at the center of triaxial elliptical galaxies [21-23]. This observation suggests that the above property (i) may appear in these galaxies, since they have high density region corresponding to core particles at their centers and low density region corresponding to halo particles surrounding them. Hence, property (i) may give a hint for explaining the relaxation process and the origin of stationally configuration shape of these galaxies, since the stochastic halo orbits that interact with core particles at the center of a triaxial galaxy may affect the equilibrium shape through the continued mixing near the center [24]. In addition, propety (ii) might help to explain the observed fractal structures and non-Gaussian velocity distributions in the interstellar medium [25], which are likely to be gravitationally virialized.

\section{ACKNOWLEDGMENTS}

We would like to thank A. Nakamichi, I. Joichi, K. Nakamura, and M. Hotta for useful discussions and comments. This work was supported partially by a Grant-in-Aid for Scientific Research Fund of the Ministry of Education, Science and Culture (Specially Promoted Research No. 08102010), and by the Waseda University Grant for Special Research Projects. 


\section{APPENDIX A: VIRIAL CONDITION FOR SGR MODEL}

We start with the nondimensional Hamiltonian of the SGR model

$$
H=\frac{1}{2} \sum_{i=1}^{N} p_{i}^{2}-\sum_{i<j}^{N} \frac{1}{\sqrt{2} N \sqrt{1-\cos \theta_{i j}+\epsilon}} .
$$

Canonical equations of motion for this system become

$$
\begin{gathered}
\frac{d \theta_{i}}{d \tau}=p_{i}, \\
\frac{d p_{i}}{d \tau}=-\sum_{j \neq i}^{N} \frac{\sin \theta_{i j}}{2 \sqrt{2} N\left(1-\cos \theta_{i j}+\epsilon\right)^{3 / 2}} .
\end{gathered}
$$

Then the second time derivative of the inertia moment of this system becomes

$$
\begin{aligned}
\frac{d^{2}}{d \tau^{2}} \sum_{i=1}^{N} \theta_{i}^{2} & =2\left(\sum_{i=1}^{N} p_{i} \frac{d \theta_{i}}{d \tau}+\sum_{i=1}^{N} \theta_{i} \frac{d p_{i}}{d \tau}\right) \\
& =2 \sum_{i=1}^{N} p_{i}^{2}-2 \sum_{i<j}^{N} \frac{\theta_{i} \sin \theta_{i j}}{\sqrt{2} N\left(1-\cos \theta_{i j}+\epsilon\right)^{3 / 2}}
\end{aligned}
$$

Taking long-time average for this equation, we obtain

$$
\left\langle\frac{d^{2}}{d \tau^{2}} \sum_{i=1}^{N} \theta_{i}^{2}\right\rangle=4 N T-2\left\langle\sum_{i<j}^{N} \frac{\theta_{i} \sin \theta_{i j}}{\sqrt{2} N\left(1-\cos \theta_{i j}+\epsilon\right)^{3 / 2}}\right\rangle .
$$

Therefore if the condition

$$
I(\tau) \equiv\left\langle\frac{d^{2}}{d \tau^{2}} \sum_{i=1}^{N} \theta_{i}^{2}\right\rangle /(N T) \ll 1
$$

is realized, then the virial relation

$$
T \approx \frac{1}{2 N}\left\langle\sum_{i<j}^{N} \frac{\theta_{i} \sin \theta_{i j}}{\sqrt{2} N\left(1-\cos \theta_{i j}+\epsilon\right)^{3 / 2}}\right\rangle
$$

holds.

By using the total potential

$$
V=-\sum_{i<j}^{N} \frac{1}{\sqrt{2} N \sqrt{1-\cos \theta_{i j}+\epsilon}},
$$

the virial relation (A6) can be expressed as

$$
T \approx \frac{1}{2 N}\left\langle\sum_{i<j}^{N} \theta_{i} \frac{\partial V}{\partial \theta_{i}}\right\rangle
$$

Note that even in the limit of $\epsilon \rightarrow 0$, the virial relation $\langle K\rangle$ $=2 N T=-\langle V\rangle / 2$ is not justified, because of the potential form (A7). However, as is shown in Sec. II, we can get the above ideal virial condition for 3D gravity within the appropriate energy range.

\section{APPENDIX B: VIRIAL CONDITION IN A $\mathcal{C}$ PHASE FOR SGR MODEL}

In $\mathcal{C}$ phase, almost all of the particles are trapped inside the core, whose size is almost $\xi$. Therefore, in this state canonical equations (A2) are approximated as

$$
\begin{gathered}
\frac{d \theta_{i}}{d \tau}=p_{i}, \\
\frac{d p_{i}}{d \tau}=-\sum_{j \neq i}^{N} \frac{\theta_{i j}}{2 \sqrt{2} N \epsilon^{3 / 2}}, \\
=-\frac{\theta_{i}}{2 \sqrt{2} \epsilon^{3 / 2}}\left(1-\frac{1}{N}\right)+\sum_{j \neq i}^{N} \frac{\theta_{j}}{2 \sqrt{2} N \epsilon^{3 / 2}},
\end{gathered}
$$

since $\left|\theta_{i j}\right| \sim O(\epsilon) \ll 1$.

In this approximation, the second time derivative of the inertia moment of this system becomes

$$
\begin{aligned}
\frac{d^{2}}{d \tau^{2}} \sum_{i} \theta_{i}^{2}= & 2 \sum_{i} p_{i}^{2}-\frac{1}{\sqrt{2} \epsilon^{3 / 2}}\left(1-\frac{1}{N}\right) \sum_{i=1}^{N} \theta_{i}^{2} \\
& +\frac{2}{\sqrt{2} N \epsilon^{3 / 2}} \sum_{i<j}^{N} \theta_{i} \theta_{j} .
\end{aligned}
$$

Taking long-time average for this relation, we obtain

$$
\begin{aligned}
\left\langle\frac{d^{2}}{d \tau^{2}} \sum_{i} \theta_{i}^{2}\right\rangle= & 4 N T-\frac{1}{\sqrt{2} \epsilon^{3 / 2}}\left(1-\frac{1}{N}\right) \sum_{i=1}^{N}\left\langle\theta_{i}^{2}\right\rangle \\
& +\frac{2}{\sqrt{2} N \epsilon^{3 / 2}} \sum_{i<j}^{N}\left\langle\theta_{i} \theta_{j}\right\rangle .
\end{aligned}
$$

Since $\theta_{i}$ and $\theta_{j}(i \neq j)$ are independent stochastic variables, the last term in Eq. (B3) vanishes. Hence, from the virial relation,

$$
T \approx \frac{1}{4 \sqrt{2} N N \epsilon^{3 / 2}}\left(1-\frac{1}{N}\right) \sum_{i=1}^{N}\left\langle\theta_{i}^{2}\right\rangle
$$

and

$$
\left\langle K_{p}\right\rangle \approx \frac{G m^{2}}{2 \sqrt{2} \epsilon^{3 / 2} R}\left(1-\frac{1}{N}\right) \sum_{i=1}^{N}\left\langle\theta_{i}^{2}\right\rangle
$$

hold. This shows that $\left\langle K_{p}\right\rangle$ in $\mathcal{C}$ phase is given by the sum of $N$ statistical elements. 
[1] W. H. Zurek, P. J. Quinn, J. K. Salmon, and M. S. Warren, Astrophys. J. 431, 559 (1994).

[2] F. Sylos Labini, M. Montuori, and L. Pietronero, Phys. Rep. 293, 61 (1998).

[3] G. de Vaucouleurs, Science 167, 1203 (1970).

[4] H. J. de Vega, N. Sánchez, and F. Combes, Phys. Rev. D 54, 6008 (1996).

[5] V. A. Antonov, Vestn. Leningr. Univ., Ser. 4: Fiz., Khim. 7, 135 (1962).

[6] D. Lynden-Bell and R. Wood, Mon. Not. R. Astron. Soc. 138, 495 (1968).

[7] I. Hachisu and D. Sugimoto, Prog. Theor. Phys. 60, 123 (1978).

[8] E. B. Aronson and C. J. Hansen, Astrophys. J. 177, 145 (1972).

[9] G. L. Camm, Mon. Not. R. Astron. Soc. 110, 305 (1950).

[10] T. Tsuchiya, N. Gouda, and T. Konishi, Phys. Rev. E 53, 2210 (1996).

[11] M. Antoni and S. Ruffo, Phys. Rev. E 52, 2361 (1995).
[12] V. Latora, A. Rapisarda, and S. Ruffo, Phys. Rev. Lett. 83, 2104 (1999).

[13] V. Latora and A. Rapisarda, Nucl. Phys. A 681, 406c (2001).

[14] T. Padmanabhan, Phys. Rep. 188, 285 (1990).

[15] P. Hertel and W. Thirring, Ann. Phys. (Paris) 63, 520 (1971).

[16] A. Compagner, C. Bruin, and A. Roelse, Phys. Rev. A 39, 5989 (1989).

[17] Y. Aizawa, K. Sato, and K. Ito, Prog. Theor. Phys. 103, 519 (2000).

[18] M. Suzuki, Phys. Lett. A 146, 319 (1990).

[19] W. Feller, An Introduction to Probability Theory and its Applications, 2nd ed. (Wiley, New York, 1966), Vol. II.

[20] K. Falconer, Fractal Geometry (Wiley, New York, 1990).

[21] T. R. Lauer et al., Astrophys. J. 110, 2622 (1995).

[22] T. R. Lauer et al., Astrophys. J. 111, 1880 (1996).

[23] K. Gebhardt et al., Astrophys. J. 112, 105 (1996).

[24] D. Merritt and T. Fridman, Astrophys. J. 460, 136 (1996).

[25] E. Falgarone, T. G. Phillips, and C. K. Walker, Astrophys. J. 378, 186 (1991). 